\title{
Reply to Andronikou and Sergot: 'point-of-care' ultrasound
}

\author{
Øystein E. Olsen ${ }^{1}$
}

Published online: 15 November 2017

(C) Springer-Verlag GmbH Germany 2017

Many thanks to Drs. Andronikou and Sergot for raising a debate about the use of the term "point-of-care" in this journal [1]. They correctly point out that the editors have been reluctant to embrace this particular expression, specifically as constituent in the construction "point-of-care ultrasound".

There are several reasons for the editorial line. The term "point-of-care" does not necessarily modify meaning. That other journals use it, does not immediately convince us that the term is meaningful. Care, as defined by the Oxford Dictionary of English, is "the provision of what is necessary for the health, welfare, maintenance, and protection of someone or something". The point of care must therefore mean the location at which this provision is executed. In the case of ultrasound, this is always where the patient is. Hence, ultrasound always takes place at the point of care, so it is unnecessary to specify this.

"Point-of-care" is used with various meanings, for example imaging performed by non-radiologists, imaging performed at bed-side, imaging performed outside a health care institution. It is not our editorial task to voice opinions about any of these practices. Our duty is to ensure that the terms used in our journal are as clearly defined as possible. Because "point-of-care" is

Øystein E. Olsen

oeolsen.pedrad@me.com

Radiology Department,

Great Ormond Street Hospital for Children NHS Foundation Trust, Great Ormond Street, London WC1N 3JH, United Kingdom equivocal, it is better replaced by a phrase that is not - or else removed.

If one accepts that there is "point-of-care imaging", then there must also be "imaging not at the point of care", and it is difficult to envisage what exactly that would be. Albeit perhaps pedantic, this suggests the need for a clearer delineation of the term.

Wherever ultrasound is performed, it remains ultrasound. This remains true whether it is performed in the ultrasound suite in a radiology department, in an emergency room, in a paediatrician's office, or in the back of a truck. Ultrasound, regardless of where it is performed and by whom, requires training, practice and expertise to be clinically helpful; and its precision, diagnostic observations, interpretations and clinical efficacy are global. If we mean "ambulatory ultrasound", then we should say so. If it is "ultrasound by non-radiologists", then that is fine too. While situation-specific descriptors would be required and much better, and there may be some validity to using the term "point-of-care" when discussing methods of service delivery, using "point-of-care ultrasound" as the name of modality, differentiated from "ultrasound", is not valid.

It is easy to agree with Drs. Andronikou and Sergot on the need to push the envelope and learn to optimise new applications for ultrasound including the examples given (pneumonia, mediastinal lymph nodes, fractures, inferior vena cava hydration status) and innumerable others. Such techniques will be performed the same regardless of the setting.

Our objective is certainly not to discredit, criticise or marginalise those outside of radiology who perform 
ultrasound. Quite the opposite; we recognise that regardless of the setting it is "ultrasound". We need to share, teach and learn from one another. Ultrasound is ubiquitous. The editors agree with Drs. Andronikou and Sergot that we should speak the same language and not be divisive, hence "ultrasound". Regardless of who does it and where it is done, let's do it well!
Compliance with ethical standards

Conflicts of interest None

\section{Reference}

1. Andronikou S, Sergot L (2017) 'Point-of-care ultrasound' — legitimate terminology. https://doi.org/10.1007/s00247-017-3978-7 